\title{
Phenotypic plasticity can facilitate adaptive evolution in gene regulatory circuits
}

\author{
Carlos Espinosa-Soto ${ }^{1,2^{*}}$, Olivier C Martin ${ }^{3}$, Andreas Wagner ${ }^{1,2,4}$
}

\begin{abstract}
Background: Many important evolutionary adaptations originate in the modification of gene regulatory circuits to produce new gene activity phenotypes. How do evolving populations sift through an astronomical number of circuits to find circuits with new adaptive phenotypes? The answer may often involve phenotypic plasticity. Phenotypic plasticity allows a genotype to produce different - alternative - phenotypes after non-genetic perturbations that include gene expression noise, environmental change, or epigenetic modification.

Results: We here analyze a well-studied model of gene regulatory circuits. A circuit's genotype encodes the regulatory interactions among circuit genes, and its phenotype corresponds to a stable gene activity pattern the circuit forms. For this model, we study how genotypes are arranged in genotype space, where the distance between two genotypes reflects the number of regulatory mutations that set those genotypes apart. Specifically, we address whether this arrangement favors adaptive evolution mediated by plasticity. We find that plasticity facilitates the origin of genotypes that produce a new phenotype in response to non-genetic perturbations. We also find that selection can then stabilize the new phenotype genetically, allowing it to become a circuit's dominant gene expression phenotype. These are generic properties of the circuits we study here.

Conclusions: Taken together, our observations suggest that phenotypic plasticity frequently facilitates the evolution of novel beneficial gene activity patterns in gene regulatory circuits.
\end{abstract}

\section{Background}

Novel adaptive phenotypes endow organisms with new means to survive and reproduce. Such new phenotypes arise through a process that involves natural selection and random genotypic change caused by mutation. Life's ability to adapt through random change is remarkable, as many man-made systems do not have this ability $[1,2]$. It is a result of how genotypic change translates into phenotypic change $[1,3]$.

Different classes of biological systems, ranging from protein and RNA molecules [4-6] to regulatory circuits [7] and genome-scale metabolic networks [8], share some similarities in how they translate genotypic change into phenotypic change. First, any genotype $G$ produces some phenotype $P$ in the absence of environmental and other perturbations. We will refer to such a 'default' phenotype as G's native phenotype (analogous to the

\footnotetext{
* Correspondence: c.espinosas@gmail.com

'University of Zurich, Dept. of Biochemistry, Bldg. Y27 Winterthurerstrasse

$190 \mathrm{CH}-8057$ Zurich, Switzerland

Full list of author information is available at the end of the article
}

native conformation of a protein). Second, in all these systems genotypes exist in vast genotype spaces. In a genotype space, the distance between two genotypes indicates the number of mutations that set those genotypes apart. Third, the set of genotypes with the same native phenotype define a "genotype network". For any two genotypes on a genotype network, there exists a sequence of small genetic changes that leads from one genotype to the other, without ever changing the native phenotype. Genotype networks are typically vast and extend far through genotype space. A population that evolves on a genotype network through mutation and selection can traverse large regions of genotype space, while the population's bulk preserves the same phenotype. While doing so, members of the population can explore different phenotypes that occur in different regions of genotype space. Because genotype networks extend far through this space, they facilitate exploration of many novel phenotypes [5,8-12]. In sum, genotype networks allow access to a wide range of new, potentially adaptive phenotypes $[13,14]$.
C Biomed Central

() 2011 Espinosa-Soto et al; licensee BioMed Central Ltd. This is an Open Access article distributed under the terms of the Creative Commons Attribution License (http://creativecommons.org/licenses/by/2.0), which permits unrestricted use, distribution, and reproduction in any medium, provided the original work is properly cited. 
Despite the existence of genotype networks, finding a specific novel phenotype through a blind evolutionary search is challenging, partly because genotype spaces are very large, partly because the fraction of advantageous novel phenotypes encountered during this search is usually small. For example, many more mutations are deleterious rather than advantageous $[15,16]$. Here we explore a phenomenon that can facilitate this search, that of phenotypic plasticity.

Phenotypic plasticity allows a genotype to produce more than one distinct phenotype [17-19]. Such alternative phenotypes are induced by non-genetic perturbations. Non-genetic perturbations influence the formation of all kinds of phenotypes, but molecular phenotypes illustrate this influence especially well. Consider protein structure phenotypes. Driven by thermal noise - a ubiquitous kind of non-genetic perturbation - the same amino acid sequence (genotype) typically folds into one main, native structure (phenotype), as well as a large spectrum of alternative structures. Several aspects of the protein's environment can influence which of these structures it forms. They include temperature, $\mathrm{pH}$, but also other proteins, such as chaperones [20] or infective proteinaceous agents (prions) [21]. The same holds for RNA molecules, where a single genotype (nucleotide sequence) can also fold into different structures $[6,22,23]$. On higher levels of organization, genes and their products interact in regulatory circuits. The genotype determines which of a circuit's genes interact; the gene activity or gene expression phenotypes resulting from these interactions are, again, influenced by nongenetic factors. For instance, a circuit's native gene expression phenotype can be altered through stochastic change - intracellular noise - in the number of regulatory molecules inside a cell [24-26]. Biotic or abiotic environmental factors can also change a gene regulatory circuit's activity pattern and the macroscopic traits it helps build [17,19,27-29].

The genotype network concept can readily accommodate the phenomenon of phenotypic plasticity [22]. Genotypes that produce a given phenotype as their native phenotype belong to the same genotype network, but each of these genotypes may also produce a spectrum of alternative phenotypes. This spectrum may differ among genotypes on the same genotype network, and not all of these genotypes may have equal plasticity. For example, one genotype may readily produce an alternative phenotype, whereas in another genotype, the same phenotype may arise only rarely, for example through an extreme and rare perturbation. In these two genotypes, the phenotype would then have high and low penetrance, respectively.

A growing body of work suggests that phenotypic plasticity strongly influences the origin of novel phenotypes
[17-19,30-44]. The earliest support comes from classic work by Waddington $[45,46]$, Schmalhausen [47] and Baldwin [48]. Waddington showed that artificial selection of a phenotype that initially appears only in a few organisms after non-genetic perturbations, can easily result in the trait's genetic determination $[46,49]$. More recently, other researchers have made the same observation for diverse traits and different species [37,38,42]. Artificial selection can thus turn an alternative into a native phenotype. In addition, many observations in wild populations suggest that in multiple cases an ancestral alternative phenotype may have facilitated the evolution of new, genetically fixed adaptive traits $[17-19,30-36,40]$. The phenotypes where plasticity may have facilitated adaptation are very diverse. They include gill surface area in cichlid fishes [33], pigmentation patterns in the crustacean Daphnia melanica [34], and head size in the snake Notechis scutatus [35], to name but a few. Despite an abundance of candidate examples, plasticity's importance for adaptive evolution is not universally accepted [50,51]. We still do not know whether existing observations from artificial selection experiments or from wild populations are rare oddities or hint at general principles of evolution $[39,43,44,52,53]$.

If important for adaptive evolution, plasticity would facilitate adaptation through a scenario such as the following (Figure 1): Consider a population in 'search' of some new superior phenotype $P_{\text {new }}$. At some point, genotypes arise that have $P_{\text {new }}$ as a low-penetrance member of their spectrum of alternative phenotypes. Such genotypes would accumulate through selection, as they occasionally produce $P_{n e w}$. Second, some mutations in these genotypes produce genotypes where $P_{\text {new }}$ has higher

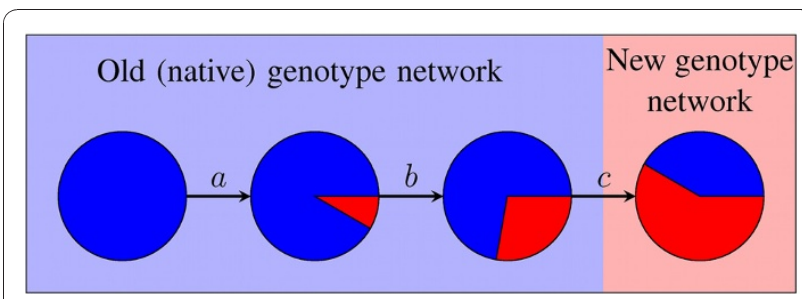

Figure 1 A plasticity-mediated evolutionary path towards a new adaptive phenotype. Each circle represents a genotype. Lines represent mutations that convert one genotype into another. Different colors represent distinct phenotypes. The same background color surrounds genotypes in the same genotype network. Colored areas within circles represent the probability that a genotype produces a particular phenotype. Blue represents an original native phenotype, and red a new beneficial phenotype. We show a sequence of mutations towards the red genotype network. First, the red phenotype arises as a low penetrance alternative phenotype (mutation a). As penetrance increases, the distance to the new genotype network decreases (b). Finally, the new phenotype has become stabilized, that is, a new genotype network has been 'discovered' (c). 
penetrance. These mutant genotypes now accumulate in the population. Finally, the population gains mutational access to $P_{\text {new }}$ 's genotype network. In this genotype network $P_{\text {new }}$ is produced as the native phenotype - without any non-genetic perturbations. Here, $P_{\text {new }}$ has become genetically stabilized.

In the above scenario, genotypes that produce a phenotype $P_{\text {new }}$ through plasticity have better chances to find $P_{\text {new }}$ 's genotype network. This scenario requires several properties of the organization of genotype space. The conditions are:

i) Finding genotypes that can produce a new alternative phenotype $P_{\text {new }}$ through plasticity must be significantly easier than finding the genotype network of $P_{\text {new }}$ (i.e. genotypes where $P_{\text {new }}$ is native).

ii) Genotypes near the genotype network of $P_{\text {new }}$ should have a tendency to produce $P_{\text {new }}$ as an alternative phenotype. Otherwise, reaching $P_{\text {new }}$ 's genotype network would not be easier from genotypes that produce $P_{\text {new }}$ through plasticity.

iii) Mutations of genotypes that produce $P_{\text {new }}$ through plasticity must often result in genotypes that can also produce $P_{\text {new }}$ through plasticity. Otherwise, an evolutionary search of $P_{\text {new }}$ 's genotype network might not be able to progress through genotypes that can all produce $P_{\text {new }}$.

iv) The higher the penetrance of $P_{\text {new }}$ is in a given genotype $G$, the easier it must be to reach $P_{\text {new }}$ 's genotype network from $G$.

Conditions i)-iii) imply that there must be sets of mutationally connected genotypes that produce $P_{\text {new }}$ as an alternative phenotype. From some of those genotypes, a single mutation must suffice to reach $P_{\text {new }}$ 's genotype network. Condition iv) implies that selection can promote genetic stabilization of $P_{\text {new }}$ through gradual increases in $P_{\text {new }}$ 's penetrance.

We here explore whether these conditions are fulfilled in a model of transcriptional regulation circuits. Such circuits produce specific gene activity patterns in different parts and developmental stages of an organism. In doing so, they have a major role in directing developmental processes $[54,55]$. Many evolutionary novelties involve changes in the gene expression patterns such circuits produce [56-59]. In the circuits we study, the genotype encodes the transcriptional regulatory interactions determined by a circuit's cis-regulatory regions. A phenotype corresponds to a circuit's stable gene activity pattern. We show that the requirements we mentioned above are fulfilled for these circuits. Our work suggests that phenotypic plasticity can facilitate adaptive evolution that involves changes in gene activity patterns of regulatory circuitry.

\section{Results}

\section{The model}

Our model represents gene regulatory circuits comprising $N$ genes. The activity of each gene in a circuit is regulated by the activity of other circuit genes. An $N$ by $N$ real-valued matrix $\mathbf{A}=\left(a_{i j}\right)$ specifies the manner in which this regulation occurs. We view this matrix as a circuit's regulatory genotype. A gene $j$ regulates the activity of another gene $i$ when $a_{i j} \neq 0$. The effect of gene $j$ on gene $i$ can be either activating $\left(a_{i j}>0\right)$ or repressing $\left(a_{i j}<0\right)$. We call two circuits neighbors (in a regulatory genotype space) if they differ in a single regulatory interaction. We use the integer variable $m$ to denote a circuit's number of regulatory interactions, i.e. the number of non-zero values in $\mathbf{A}$; we use the real number $c$ to denote a circuit's interaction density, that is, its number of interactions $m$ divided by the maximally possible number of interactions $N^{2}$. We describe the activity state of the circuit at time $t$ with a vector $s_{t}=\left(s_{t}^{(1)}, \ldots, s_{t}^{(N)}\right)$. In our model, cross-regulatory interactions among circuit genes and the circuit's activity state at time $t$ determine the circuit's activity state after a time-step of length $\tau$ as follows:

$$
s_{t+\tau}^{(i)}=\sigma\left[\sum_{j=1}^{N} a_{i j} s_{t}^{(j)}\right]
$$

where $\sigma(x)$ equals -1 when $x<0$, it equals +1 when $x$ $>0$, and it equals 0 when $x=0$.

Despite this model's simplicity, it has been successfully used to study various aspects of the evolution of gene regulatory circuits, such as the evolution of robustness, of modularity, and of pattern formation [7,11,60-66]. Variants of the model have also proven useful to model developmental processes in plants and animals $[67,68]$.

We here consider circuits that attain a stable gene activity pattern $s_{\infty}$ when their dynamics start from an initial gene activity pattern $s_{0}$. Such an initial state is determined by factors outside the circuit, be they genes 'upstream' of the circuit, maternal regulators, signals from neighboring cells or environmental factors. We refer to a stable gene activity pattern $s_{\infty}$ as a gene activity phenotype. As in previous research [7], we do not analyze circuits that fail to produce a stable activity pattern.

Circuit genotypes with the same gene activity phenotype form vast genotype networks in a space of regulatory circuits [7]. Throughout this paper, we consider circuits in a given genotype network, that is, they attain a given gene activity phenotype $s_{\infty}^{\text {native }}$ from a given initial gene activity state $\mathrm{s}_{0}$ through the circuit's dynamics. We refer to this genotype network as the 
'native genotype network' and to $s_{\infty}^{\text {native }}$ as the 'native phenotype'. The activity state $s_{0}$ is the gene activity state from which the system starts its dynamics in the absence of non-genetic perturbations. We note that all properties of genotype network organization relevant to us depend on the fraction $d$ of gene activity differences between $s_{0}$ and $s_{\infty}^{\text {native }}$, and not on the identity of these activity patterns [7].

We study two kinds of perturbations. The first is a mutation of a circuit's regulatory genotype. A mutation changes an interaction by altering a value of $a_{i j}$ in a circuit's matrix A. Some mutations can cause a circuit to produce a phenotype different from $s_{\infty}^{\text {native }}$. The second kind of perturbation has a non-genetic origin and affects the initial gene activity pattern $\mathrm{s}_{0}$. Such a perturbation could result, for example, from intracellular noise, from environmental fluctuations, or from disturbances in the activity of genes upstream of the circuit. For example, intracellular noise can create gene expression heterogeneity in clonal populations [24-26], just as exposure to some environmental factors can induce major gene expression changes in different organismal lineages [27-29], and impairing the activity of pair-rule genes upstream of the segment-polarity gene circuit in fruit flies can change the expression pattern of genes in this circuit $[69,70]$. Such perturbations can alter developmental trajectories, and result in new gene activity phenotypes different from $s_{\infty}^{\text {native }}$.

We call the phenotypes that a circuit genotype $G$ produces after non-genetic perturbations and that are different from $G$ 's native phenotype 'alternative phenotypes'. Some alternative phenotypes may be detrimental, but others may be beneficial $[40,71,72]$. An alternative phenotype has a low (high) penetrance if the likelihood that $G$ produces it after a random perturbation in the initial condition is low (high).

\section{Finding new alternative phenotypes is easier than finding new native phenotypes}

We first asked whether mutation-driven exploration of a genotype network can find new alternative phenotypes more easily than new genotype networks (native phenotypes), as required by condition i) in the introduction. To answer this question, we allowed an ensemble of $5 \times$ $10^{3}$ circuits to drift randomly on a genotype network by changing one regulatory interaction at a time, while preserving the circuits' native phenotype. During this process, we recorded two observables. The first was the cumulative number of new phenotypes that circuits could produce after each possible single gene-activity modifications of $s_{0}$ (i.e. non-genetic perturbations). That is, after each mutation, we determined those alternative phenotypes that a mutated genotype could produce but that previous genotypes had not been able to produce, and appended these alternative phenotypes to a growing list of such phenotypes. The second observable was the cumulative number of new phenotypes that the circuits explored exclusively through mutation. Every time a mutation caused a change in a circuit's native phenotype, we recorded the new phenotype, before replacing the circuit by its parent in the original native genotype network. We appended these new phenotypes to a growing list of phenotypes that had not been encountered through previous mutations. In this analysis, we found that plasticity allows a faster exploration of new phenotypes than mutation alone. Figure 2 shows that after each circuit in the ensemble had experienced 500 mutations, plasticity had explored more than twice as many phenotypes as mutation. This figure averages results for 200 independent ensembles of circuits with $N=16$ genes, interaction density $c \approx 0.35$, and a fraction $d$ of gene activity differences between $s_{0}$ and $s_{\infty}^{\text {native }}$ equal to 0.125 . Circuits with different values of these parameters also have faster access to new phenotypes through plasticity than through mutation alone (Additional file 1: Figure S1).

\section{Mutations and non-genetic perturbations produce similar sets of phenotypes}

If phenotypic plasticity facilitates the discovery of new genotype networks, then mutations and non-genetic change should often produce similar or identical new phenotypes (condition ii) in the introduction). Otherwise, genotypes that produce an alternative phenotype after non-genetic perturbations would not have preferential mutational access to that phenotype's genotype network. In other words, if condition ii) did not hold,

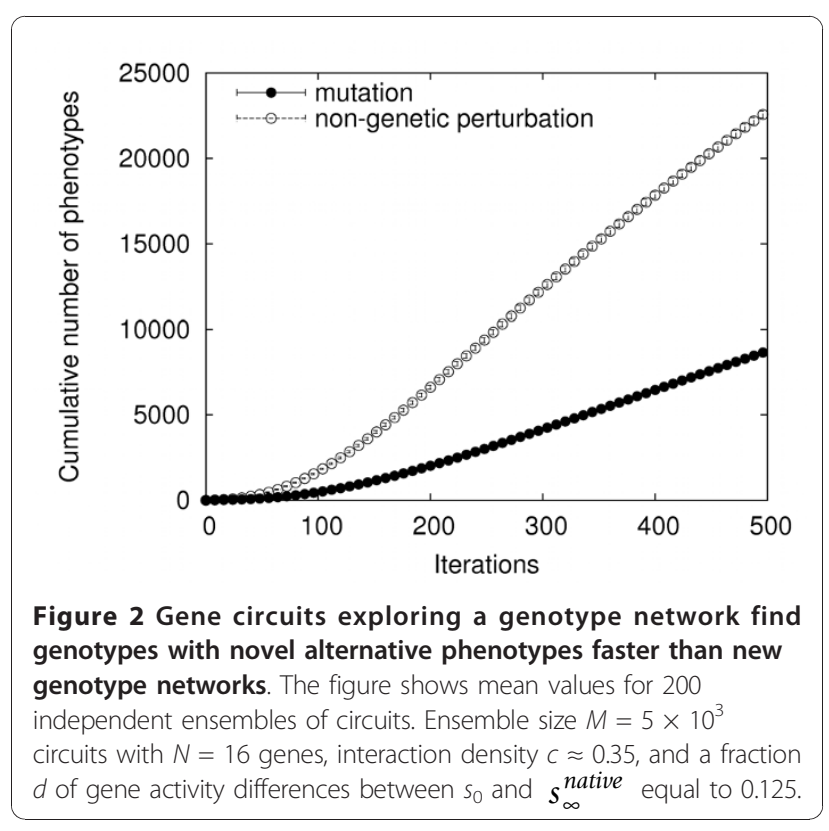


mutations would not be able to turn an alternative phenotype into a native phenotype that is produced even in the absence of non-genetic perturbations. To find out whether this is the case, we sampled random genotypes from a given genotype network. All the genotypes on this network produce the same phenotype $s_{\infty}^{\text {native }}$ from $s_{0}$. We defined the following sets for each genotype $G$ in the sample: $P_{\mu}$ is the set of new phenotypes that mutations in $G$ can create; $P_{s_{0}}$ is the set of alternative phenotypes that perturbation of each gene in the initial condition $s_{0}$ can create. To quantify the similarity between both sets, we defined the index $C$ as the size of the intersection between both sets divided by its maximally possible value for sets of the same size $\left(C=\frac{\left|P_{\mu} \cap P_{s_{0}}\right|}{\min \left(\left|P_{\mu}\right|,\left|P_{s_{0}}\right|\right)}\right) \cdot C$ ranges from zero, if mutation and non-genetic perturbations produce always different phenotypes, to one, if $P_{\mu}$ and $P_{s_{0}}$ fully overlap. We also defined an index $C^{\text {rand }}$, which estimates how similar randomly chosen sets of phenotypes would be (see Methods). By assessing $C$, we found that mutations and non-genetic perturbations produce the same phenotypes more often than expected by chance (Additional file 2: Figure S2). This observation holds for all combinations of circuit sizes $N$, interaction densities $c$, and distances $d$ between $s_{0}$ and $s_{\infty}^{\text {native }}$ we examined (Table 1; Wilcoxon signed-rank test; $p<2.2 \times 10^{-16}$ in all cases). Thus,

Table 1 Mutations and perturbations in the initial condition s0 produce the same phenotypes more often than expected by chance

\begin{tabular}{|c|c|c|c|c|c|}
\hline$N$ & $c$ & $d$ & Mean $C \pm$ S.E. & $p$-value & Sample size ${ }^{a}$ \\
\hline \multirow[t]{6}{*}{8} & 0.4 & 0.125 & $0.528 \pm 0.006$ & $<2.2 \times 10^{-16}$ & 5485 \\
\hline & & 0.25 & $0.496 \pm 0.006$ & $<2.2 \times 10^{-16}$ & 5905 \\
\hline & & 0.5 & $0.557 \pm 0.005$ & $<2.2 \times 10^{-16}$ & 7269 \\
\hline & 0.3 & 0.125 & $0.442 \pm 0.006$ & $<2.2 \times 10^{-16}$ & 5885 \\
\hline & & 0.25 & $0.398 \pm 0.006$ & $<2.2 \times 10^{-16}$ & 6050 \\
\hline & & 0.5 & $0.349 \pm 0.005$ & $<2.2 \times 10^{-16}$ & 7144 \\
\hline \multirow[t]{9}{*}{20} & 0.3 & 0.1 & $0.712 \pm 0.006$ & $<2.2 \times 10^{-16}$ & 5247 \\
\hline & & 0.25 & $0.8 \pm 0.005$ & $<2.2 \times 10^{-16}$ & 6432 \\
\hline & & 0.5 & $0.885 \pm 0.003$ & $<2.2 \times 10^{-16}$ & 8055 \\
\hline & 0.2 & 0.1 & $0.63 \pm 0.006$ & $<2.2 \times 10^{-16}$ & 5318 \\
\hline & & 0.25 & $0.645 \pm 0.006$ & $<2.2 \times 10^{-16}$ & 5699 \\
\hline & & 0.5 & $0.711 \pm 0.005$ & $<2.2 \times 10^{-16}$ & 7102 \\
\hline & 0.1 & 0.1 & $0.47 \pm 0.006$ & $<2.2 \times 10^{-16}$ & 6036 \\
\hline & & 0.25 & $0.408 \pm 0.006$ & $<2.2 \times 10^{-16}$ & 6058 \\
\hline & & 0.5 & $0.364 \pm 0.005$ & $<2.2 \times 10^{-16}$ & 6776 \\
\hline
\end{tabular}

$\mathrm{C}>\mathrm{C}^{\text {rand }}$, according to a Wilcoxon signed-rank test. In this and all other tables, $N$ refers to the number of genes in a circuit, $c$ refers to the circuit's interaction density, and $d$ refers to the fraction of gene activity differences between the unperturbed initial condition $s_{0}$ and the native phenotype $s_{\infty}^{\text {native }}$.

${ }^{a}$ Even though we sampled $10^{4}$ genotypes for each genotype network, we discarded genotypes in which either $P_{\mu}$ or $P_{s_{0}}$ was empty. genotypes that require a single mutation to reach the genotype network of a phenotype $P_{\text {new }}$ also tend to produce $P_{\text {new }}$ as an alternative phenotype.

\section{Similar circuits produce a similar spectrum of alternative phenotypes}

Genotypes producing a new phenotype $s_{\infty}^{\text {new }}$ through plasticity, but that are not themselves neighbors of $s_{\infty}^{\text {new }}$ 's genotype network may still aid in the genetic stabilization of $s_{\infty}^{\text {new }}$. This could occur if neighboring genotypes typically produce the same alternative phenotypes [condition iii)]. In this case, mutations that preserve $s_{\infty}^{\text {new }}$ as an alternative phenotype can make it easier to reach $s_{\infty}^{\text {new }}$ 's genotype network.

Motivated by these considerations, we asked whether similar circuits produce similar sets of alternative phenotypes. We use the symbol $P_{s_{0}}$ to refer to the set of alternative phenotypes that a circuit genotype $G$ produces after non-genetic perturbations. $P_{s_{0}, k}$ refers to the set of alternative phenotypes that a genotype differing from $G$ in $k$ regulatory interactions, but residing on the same genotype network produces. We define the index $C_{k}=\frac{\left|P_{s_{0}} \cap P_{s_{0}, k}\right|}{\min \left(\left|P_{s_{0}}\right|,\left|P_{s_{0}, k}\right|\right)}$. It varies from zero to one as the two sets of alternative phenotypes range from completely disjoint to fully overlapping.

Similar circuit genotypes produce similar sets of alternative phenotypes (Figure 3). Genotypes that differ in a

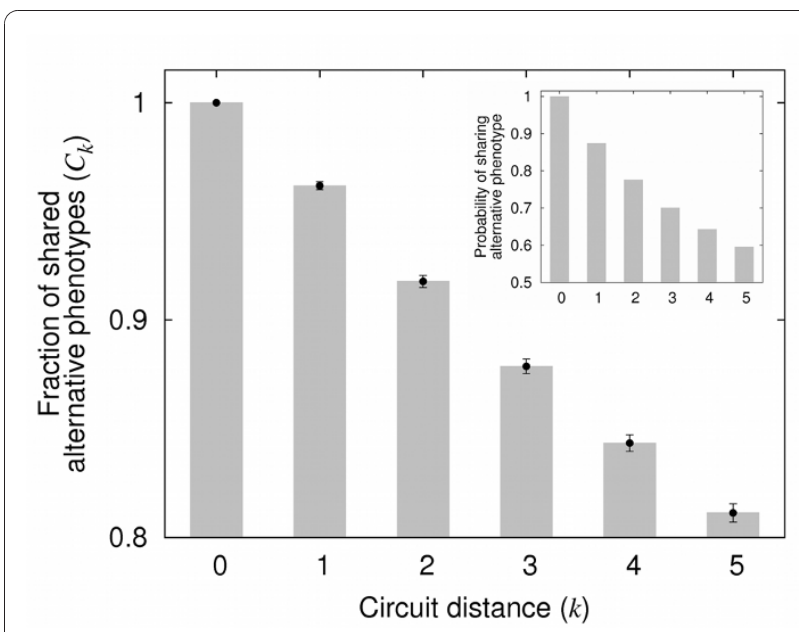

Figure 3 The more similar two circuit genotypes are, the higher the overlap between their sets of alternative phenotypes. The number $k$ represents the distance (in the number of differing regulatory interactions) between two circuits. $C_{k}$ measures the similarity between the sets of alternative phenotypes produced by the two circuits. The plot shows mean values and the length of error bars represents one standard error. Spearman's $\rho=-0.248 ; p<2.2 \times 10^{-16}$. The inset shows that the fraction of genotype pairs that share a given alternative phenotype decreases with $k$. Data are based on $10^{4}$ randomly sampled focal circuits with $N=20, c \approx 0.2$, and $d=0.1$. 
single mutation share, on average, more than $93 \%$ of their alternative phenotypes $\left(C_{1}>0.93\right)$. High similarity in alternative phenotypes also holds after variation on values of $N, c$ and $d$ (Table 2). Figure 3 also illustrates how $C_{k}$ decreases as two circuits diverge. Circuit distance $k$ and $C_{k}$ are negatively associated for all values of $N, c$ and $d$ that we examined (Table 2; Spearman's $\rho ; p$ $<2.210^{-16}$ in all cases).

In addition, for each of $10^{4}$ circuits on the same genotype network, we picked randomly one of the circuit's alternative phenotypes. We asked whether genotypes in the same genotype network but differing from the focal genotype in $k$ regulatory interactions also produced the alternative phenotype through plasticity. More than half

\begin{tabular}{|c|c|c|c|c|c|}
\hline$N$ & $c$ & $d$ & $\begin{array}{l}\text { Mean } C_{1} \pm \text { S.E. } \\
\left(\text { Sample size }{ }^{a}\right)\end{array}$ & $\begin{array}{c}\text { Spearman's } \\
\rho\end{array}$ & $p$-value \\
\hline \multirow[t]{6}{*}{8} & 0.4 & 0.125 & $0.95 \pm 0.002(9124)$ & -0.279 & $<\underset{16}{2.2 \times 10^{-}}$ \\
\hline & & 0.25 & $0.956 \pm 0.002(9248)$ & -0.247 & $<\underset{16}{2.2} \times 10^{-}$ \\
\hline & & 0.5 & $0.968 \pm 0.002(9499)$ & -0.193 & $<\underset{16}{2.2 \times 10^{-}}$ \\
\hline & 0.3 & 0.125 & $0.933 \pm 0.002(9408)$ & -0.295 & $<\underset{16}{2.2 \times 10^{-}}$ \\
\hline & & 0.25 & $0.94 \pm 0.002$ & -0.27 & $<\underset{16}{2.2 \times 10^{-}}$ \\
\hline & & 0.5 & $0.963 \pm 0.002(9649)$ & -0.206 & $<\underset{16}{2.2 \times 10^{-}}$ \\
\hline \multirow[t]{9}{*}{20} & 0.3 & 0.1 & $0.968 \pm 0.002(8712)$ & -0.237 & $<\underset{16}{2.2 \times 10^{-}}$ \\
\hline & & 0.25 & $0.97 \pm 0.002(8910)$ & -0.201 & $<\underset{16}{2.2 \times 10^{-}}$ \\
\hline & & 0.5 & $0.98 \pm 0.001$ (9403) & -0.153 & $<\underset{16}{2.2} \times 10^{-}$ \\
\hline & 0.2 & 0.1 & $0.962 \pm 0.002(9216)$ & -0.248 & $<\underset{16}{2.2 \times 10^{-}}$ \\
\hline & & 0.25 & $0.965 \pm 0.002(9179)$ & -0.214 & $<\underset{16}{2.2 \times 10^{-}}$ \\
\hline & & 0.5 & $0.977 \pm 0.001(9440)$ & -0.178 & $<\underset{16}{2.2 \times 10^{-}}$ \\
\hline & 0.1 & 0.1 & $0.948 \pm 0.002(9675)$ & -0.255 & $<\underset{16}{2.2} \times 10^{-}$ \\
\hline & & 0.25 & $0.957 \pm 0.002(9696)$ & -0.237 & $<\underset{16}{2.2} \times 10^{-}$ \\
\hline & & 0.5 & $0.966 \pm 0.002(9748)$ & -0.189 & $<\underset{16}{2.2} \times 10^{-}$ \\
\hline
\end{tabular}

This overlap decreases with the genetic distance between the circuits, according to Spearman's $\rho$, a non-parametric rank correlation coefficient [76] $C_{1}$ is the overlap in the sets of alternative phenotypes of two genotypes that differ in a single regulatory interaction.

${ }^{a}$ We sampled $10^{4}$ independent genotypes for each parameter combination, but we discarded genotypes that differ in $k$ interactions where $P_{s 0, k}$ was empty. of the genotypes that differ from the focal genotype by five or fewer mutations also produced the same alternative phenotype (inset in Figure 3 and Table 3). For all values of $N, c$ and $d$ we examined, the probability that two genotypes produce the same alternative phenotype was above 0.8 for genotypes that differ in a single regulatory interaction (Table 3). This probability decreases with the number $k$ of regulatory interactions in which two circuits differ (Figure 3 and Table 3). In sum, similar circuits have better odds to produce the same alternative phenotype. Thus, genotypes that produce an alternative phenotype $s_{\infty}^{\text {new }}$ but that are not neighbors of $s_{\infty}^{\text {new }}$ 's genotype network can have indirect mutational access to this network. Other genotypes that can produce $s_{\infty}^{\text {new }}$ as an alternative phenotype may provide this access, enabling genetic stabilization of the new phenotype.

\section{Genetic distance to a new genotype network is negatively correlated to a phenotype's penetrance}

Thus far, we demonstrated that conditions i) through iii) of our evolutionary scenario hold. We now turn to condition iv). This condition requires that circuits with a high penetrance alternative phenotype $P_{\text {new }}$ have preferential (mutational) access to $P_{\text {new }}$ 's genotype network, where this phenotype is native. We next show in several complementary ways that this is the case.

We quantified the penetrance of a given phenotype as the fraction of different single-gene expression perturbations in a circuit's initial state that produce this phenotype. We then assessed whether a phenotype's penetrance is linked to a circuit's proximity to a new genotype network, as follows (Figure 4a). We first chose a genotype $G$ at random among all genotypes in a predetermined genotype network. Second, we determined $G$ 's alternative phenotypes and their penetrance. Third, we chose one of the alternative phenotypes at random, regardless of its penetrance, and called it $s_{\infty}^{\text {new }}$. Fourth, we estimated the distance of $G$ to the genotype network of $s_{\infty}^{\text {new }}$ (see Methods). We repeated this procedure for $10^{4}$ genotypes for each combination of values of $N, c$ and $d$ that we examined.

Figure $4 \mathrm{~b}$ illustrates our findings. The horizontal axis shows a phenotype's penetrance, and the vertical axis the distance to the new genotype network. The area of each circle reflects the number of genotypes in each penetrance/mutational distance category. Starting from a genotype that produces an alternative phenotype $s_{\infty}^{\text {new }}$, one to two mutations are generally sufficient to reach $s_{\infty}^{\text {new }}$ 's genotype network. Figure $4 \mathrm{~b}$ also shows that distance to a new genotype network decreases with increasing penetrance. The inset focuses on the fraction of genotypes that require a single mutation to reach the new genotype network. This fraction increases - new 
Table 3 The probability that two circuit genotypes share an alternative phenotype decreases with their genotypic distance

\begin{tabular}{|c|c|c|c|c|c|c|c|}
\hline \multirow[t]{2}{*}{$N$} & \multirow[t]{2}{*}{$c$} & \multirow[t]{2}{*}{$d$} & \multicolumn{5}{|c|}{ Fraction of genotypes that share a specific phenotype after $k$ mutations. } \\
\hline & & & $k=1$ & $k=2$ & $k=3$ & $k=4$ & $k=5$ \\
\hline \multirow[t]{6}{*}{8} & 0.4 & 0.125 & 0.854 & 0.749 & 0.664 & 0.593 & 0.546 \\
\hline & & 0.25 & 0.874 & 0.777 & 0.706 & 0.645 & 0.6 \\
\hline & & 0.5 & 0.911 & 0.839 & 0.786 & 0.748 & 0.713 \\
\hline & 0.3 & 0.125 & 0.869 & 0.768 & 0.691 & 0.624 & 0.568 \\
\hline & & 0.25 & 0.882 & 0.787 & 0.716 & 0.657 & 0.606 \\
\hline & & 0.5 & 0.925 & 0.863 & 0.809 & 0.765 & 0.727 \\
\hline \multirow[t]{9}{*}{20} & 0.3 & 0.1 & 0.826 & 0.718 & 0.644 & 0.59 & 0.546 \\
\hline & & 0.25 & 0.85 & 0.753 & 0.687 & 0.646 & 0.619 \\
\hline & & 0.5 & 0.907 & 0.849 & 0.804 & 0.774 & 0.75 \\
\hline & 0.2 & 0.1 & 0.874 & 0.776 & 0.701 & 0.643 & 0.596 \\
\hline & & 0.25 & 0.877 & 0.782 & 0.719 & 0.663 & 0.617 \\
\hline & & 0.5 & 0.913 & 0.85 & 0.799 & 0.762 & 0.73 \\
\hline & 0.1 & 0.1 & 0.913 & 0.848 & 0.784 & 0.728 & 0.676 \\
\hline & & 0.25 & 0.924 & 0.857 & 0.797 & 0.743 & 0.701 \\
\hline & & 0.5 & 0.939 & 0.891 & 0.849 & 0.811 & 0.779 \\
\hline
\end{tabular}

\section{$\mathbf{a}$}

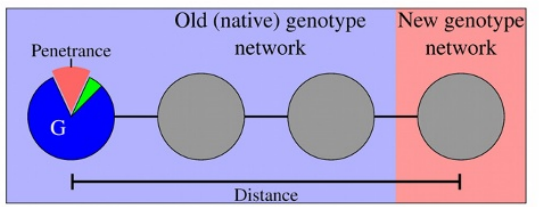

b

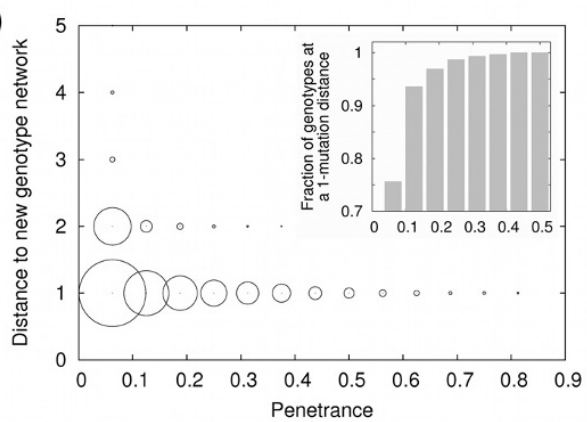

Figure 4 Genetic distance to a genotype network is negatively correlated with a phenotype's penetrance. (a) The leftmost circle represents a genotype $G$, where an alternative phenotype $s_{\infty}^{\text {new }}$ has a given penetrance (red sector). We quantified the penetrance of $s_{\infty}^{\text {new }}$ in $G$ as the fraction of single-gene perturbations of the initial condition $s 0$ that produce $s_{\infty}^{\text {new }}$. We determined genetic distance as the smallest number of mutations required to reach $s_{\infty}^{\text {new }}$ 's genotype network (rightmost circle). (b) The horizontal axis shows the penetrance of a phenotype $s_{\infty}^{\text {new }}$. The vertical axis shows the distance to $s_{\infty}^{\text {new 's }}$ genotype network. A circle's area is proportional to the number of data points in each penetrance and mutational distance category. The panel is based on $10^{4}$ circuit genotypes with $N=16$ genes, $c \approx 0.35$, and $d=0.25$. Penetrance and genetic distance to the new genotype network are negatively associated (Spearman's $\rho=-0.293 ; p<2.2 \times 10^{-}$ $\left.{ }^{16}\right)$. The inset shows that the fraction of genotypes that are neighbors of the new genotype network increases with penetrance. genotype networks become easier to reach - as the penetrance of alternative phenotypes increases. The distance to a new genotype network decreases with increasing penetrance in all parameter combinations that we examined (Table 4; Spearman's $\rho$; $p<2.2 \times 10^{-}$ 16 in all cases). The same holds when we consider alternative phenotypes produced by the perturbation of two instead of just one gene in the initial condition (Additional file 3: Analysis S1).

We performed two additional complementary analyses, that we present in the Additional files. In the first analysis we found that genotypes that produce an alternative phenotype with high penetrance have more mutational paths towards the genotype network of that phenotype (Additional file 4: Analysis S2). In the second analysis we compared two kinds of sequences of mutations: those that increase the penetrance of an alternative phenotype $s_{\infty}^{\text {new }}$ and those that merely preserve the alternative phenotype $s_{\infty}^{\text {new }}$, regardless of its penetrance. We show that the former kind of mutations facilitates the arrival to $s_{\infty}^{\text {new }}$ 's genotype network (Additional file 5: Analysis S3). Taken together, the observations in this section show that the genotype network of a new phenotype is closer and easier to reach from genotypes where a new phenotype has high penetrance.

\section{Plasticity accelerates the discovery of new genotype networks}

Thus far, all our results support that plasticity facilitates discovery and stabilization of new phenotypes. We next asked more directly whether this is the case, by analyzing 
Table 4 Genetic distance to a new genotype network decreases with increasing penetrance

\begin{tabular}{ccccc}
\hline $\boldsymbol{N}$ & $\boldsymbol{c}$ & $\boldsymbol{d}$ & Spearman's $\boldsymbol{\rho}$ & $\boldsymbol{p}$-value \\
\hline 8 & 0.4 & 0.25 & -0.268 & $<2.2 \times 10^{-16}$ \\
\cline { 3 - 5 } & & 0.125 & -0.211 & $<2.2 \times 10^{-16}$ \\
\cline { 2 - 5 } & 0.3 & 0.25 & -0.218 & $<2.2 \times 10^{-16}$ \\
\hline \multirow{2}{*}{16} & 0.35 & 0.25 & -0.293 & $<2.2 \times 10^{-16}$ \\
\cline { 2 - 5 } & 0.25 & 0.125 & -0.263 & $<2.2 \times 10^{-16}$ \\
\cline { 3 - 5 } & & 0.25 & -0.306 & $<2.2 \times 10^{-16}$ \\
\hline \multirow{2}{*}{20} & 0.3 & 0.25 & -0.26 & $<2.2 \times 10^{-16}$ \\
\cline { 2 - 5 } & 0.2 & 0.1 & -0.269 & $<2.2 \times 10^{-16}$ \\
\cline { 2 - 5 } & & 0.25 & -0.33 & $<2.2 \times 10^{-16}$ \\
\cline { 2 - 5 } & & 0.5 & -0.4 & $<2.2 \times 10^{-16}$ \\
\cline { 2 - 5 } & 0.1 & 0.25 & -0.23 & $<2.2 \times 10^{-16}$ \\
\hline
\end{tabular}

Data for each parameter combination is based on $10^{4}$ different circuits and on the non-parametric rank correlation coefficient Spearman's $\rho$ [76].

populations of circuits subject to repeated cycles of mutation and selection.

We started by establishing a population of $M=10^{3}$ identical circuits. These circuits produce a phenotype $s_{\infty}^{\text {native }}$ from an initial state $s_{0}$. We assigned individuals with this native phenotype a fitness (survival probability) $\omega^{\text {native }}<1$. Then, we chose a random gene activity phenotype $s_{\infty}^{\text {new }}$ as the target of an evolutionary search. We assigned individuals adopting $s_{\infty}^{\text {new }}$ a fitness of $\omega^{\text {new }}=1$. Starting from our initial population, we then carried out two parallel evolutionary simulations. In both we changed the population through repeated generations of replication, mutation and selection (see Methods).

In the first kind of simulation, we randomly perturbed each circuit's initial state every generation. We did so by perturbing each gene's initial activity state with a probability $\alpha$. Then, we followed the circuit's gene activity dynamics, until the circuit attained its stable gene activity phenotype. We note that two circuits with the same genotype may produce different phenotypes because of these perturbations. We kept the rate of perturbation $\alpha$ low enough so that in most individuals the initial condition remained unperturbed. In the second, parallel 'control' simulation, we never perturbed the initial condition. Here, individuals needed to 'discover' the novel phenotype exclusively through mutation.

In both simulations we recorded two quantities. The first is the time (in generations) until the first individual in the population 'discovers' the genotype network of $s_{\infty}^{\text {new }}$. We call these times $t_{\text {", plast }}$ and $t^{\prime \prime, \text { control }}$ for the simulations with plasticity and the control simulations. The second is the time, from either $t^{*}$, plast or $t$, control, until at least one quarter of the population lies in the new genotype network. We call these times $t_{0.25}$, plast and $t_{0.25}$, control, respectively.
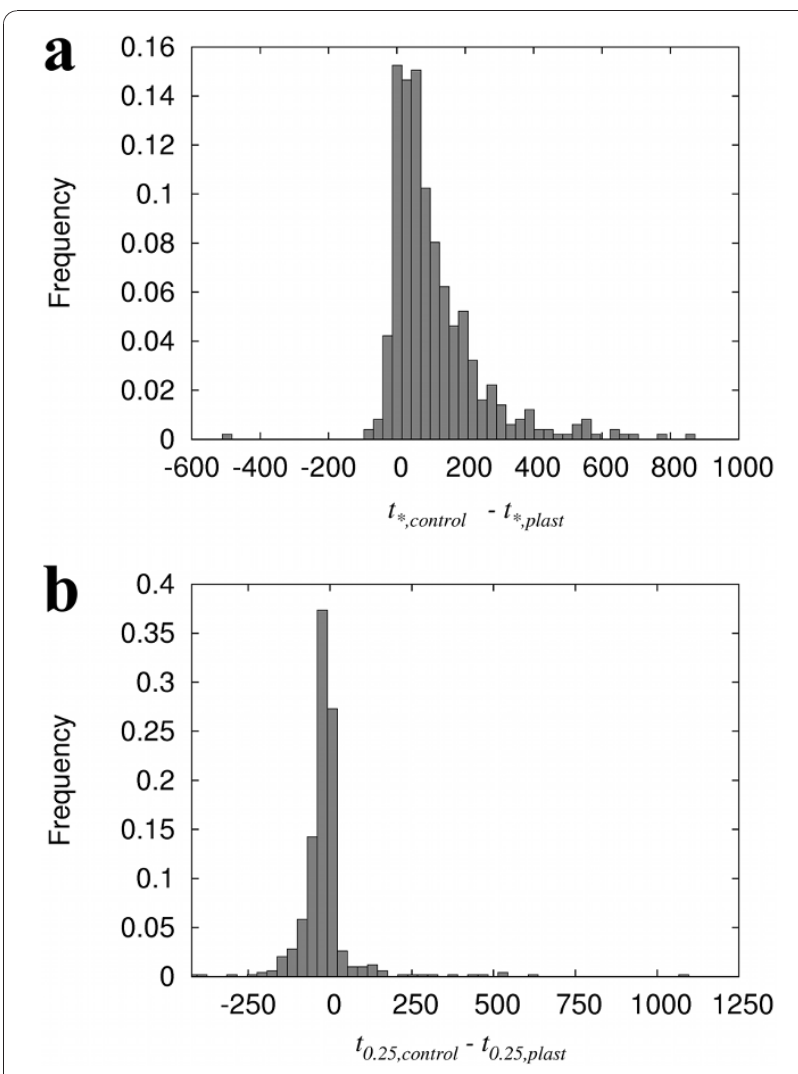

Figure 5 Plasticity affects the speed of novel phenotype acquisition. (a) Populations find a novel genotype network faster when plasticity is allowed. The symbol $t_{*}$, plast refers to the number of generations that a population of circuits needs to discover a specific genotype network when we allow plasticity. The symbol $t_{*}$, control refers to the same number, but for populations without plasticity. Wilcoxon signed-rank test; $p<2.2 \times 10^{-16}$. (b) Plasticity slows the accumulation of individuals in the new genotype network. The symbol $t_{0.25, \text { plast }}$ stands for the number of generations that a population in which we allow plasticity needs to have at least 25 percent of its circuits in the new genotype network (after its discovery by a single individual); $t_{0.25, \text { control }}$ corresponds to the same number but without plastic phenotypes. Wilcoxon signed-rank test; $p<2.2 \times 10^{-16}$. Both panels include data from 498 independent pairs of populations. Parameter values for both panels: $N=8, c \approx 0.4, d=0.25, \mu=0.5, \alpha=0.05, M=1000, \omega^{\text {native }}=0.5$.

Figure 5a illustrates that populations in which we allow plasticity usually discover the new genotype network - a genetically determined novel phenotype - faster than control populations $\left(t_{*, \text { plast }}<t_{\text {", control }}\right)$. This observation holds after variation in values of $N, c$ and $d$ : with plasticity, the time to discovery of a new genotype network is significantly lower in all cases (Table 5; Wilcoxon signed-rank test). We also varied the rate of perturbation per gene in $s_{0}$, the fitness of the native phenotype $\omega^{\text {native }}$, and the population size $M$. Nearly all such variations yielded a significantly shorter discovery time when we allowed phenotypic plasticity (Table 6; 
Table 5 Plasticity accelerates the discovery of a new optimal genotype network

\begin{tabular}{|c|c|c|c|c|c|c|}
\hline$N$ & c & $d$ & $\begin{array}{c}\text { Sample } \\
\text { size }\end{array}$ & $\begin{array}{c}\text { Mean } t_{*} \\
\text { control }\end{array}$ & Mean $t_{*}$, plast & $p$-value \\
\hline \multirow[t]{6}{*}{8} & 0.4 & 0.125 & 498 & 164.93 & 54.45 & $<2.2 \times 10^{-16}$ \\
\hline & & 0.25 & 498 & 160.3 & 52.86 & $<2.2 \times 10^{-16}$ \\
\hline & & 0.5 & 497 & 167.58 & 46.95 & $<2.2 \times 10^{-16}$ \\
\hline & 0.3 & 0.125 & 495 & 104.92 & 42.82 & $<2.2 \times 10^{-16}$ \\
\hline & & 0.25 & 498 & 103.84 & 38.03 & $<2.2 \times 10^{-16}$ \\
\hline & & 0.5 & 498 & 136.33 & 40.53 & $<2.2 \times 10^{-16}$ \\
\hline \multirow[t]{6}{*}{16} & 0.25 & 0.125 & 420 & 2744.8 & 2319.81 & 0.00059 \\
\hline & & 0.25 & 435 & 2792.44 & 2299.51 & $3.9 \times 10^{-5}$ \\
\hline & & 0.5 & 421 & 2832.02 & 2173.54 & $2.2 \times 10^{-7}$ \\
\hline & 0.2 & 0.125 & 464 & 2510.68 & 1700.95 & $1.4 \times 10^{-11}$ \\
\hline & & 0.25 & 468 & 2483.48 & 1867.23 & $1.2 \times 10^{-6}$ \\
\hline & & 0.5 & 473 & 2400.1 & 1758.22 & $1.4 \times 10^{-7}$ \\
\hline 20 & 0.2 & 0.25 & 154 & 3961.17 & 3303.32 & 0.006869 \\
\hline
\end{tabular}

The number of generations that a population takes to 'discover' a circuit in a new genotype network is significantly lower when we allow plasticity ( $t_{*}$, plast $<t_{*}$, control), according to a Wilcoxon signed-rank test.

The value of $d$ is that of the old genotype network. We analyzed 500 pairs of evolving populations for each combination of $N, c$ and $d$. We discarded population pairs in which any of the populations had not reached the new genotype network by the end of the simulation $\left(t=10^{4}\right)$. Thus, our actual sample size was lower than 500 populations. The probability $\alpha$ ofgene-activity perturbation in $s_{0}$ equaled 0.05 per gene when $N=8,0.025$ when $N=16$, and 0.02 when $N=20$. Population size $M=1000 ; \mu=0.5 ; \omega^{\text {native }}=0.5$.
Wilcoxon signed-rank test). In sum, plasticity robustly accelerated the discovery of new, genetically stable phenotypes.

In contrast to these observations, the time from the discovery of a new genotype network until 25 percent of a population occupied this genotype network was longer for populations in which we allowed plasticity $\left(t_{0.25 \text {,control }}<t_{0.25 \text {,plast }} ;\right.$ Figure $\left.5 \mathrm{~b}\right)$. This difference, albeit small, is statistically significant for all combinations of values for $N, c$ and $d$ that we tested (Table 7). Thus, plasticity can slow the rate at which individuals with a genetically determined new phenotype increase in frequency. In sum, the shorter discovery time of a new genotype network associated with phenotypic plasticity is followed by a slower transition into this new network. The small plasticity-dependent delay that we observe in our work coincides with predictions of a previously published theoretical study [29]. In that study, the delay arises because of a decreased selection differential associated with alleles that have conditional fitness effects.

\section{Discussion}

The model of gene regulatory circuits we use here is a coarse, abstract representation of the real complexity of such circuits. This is its main limitation and, at the same time, its strength. It allows us to analyze millions of circuits, their native phenotypes, and any alternative

Table 6 For nearly all combinations of parameter values, plasticity accelerates the discovery of a new optimal genotype network

\begin{tabular}{|c|c|c|c|c|c|c|c|}
\hline$N$ & $\alpha$ & $\omega^{\text {native }}$ & $M$ & Sample size & Mean $t_{*}$, control & Mean $t_{*}$, plast & $p$-value \\
\hline \multirow[t]{8}{*}{8} & 0.02 & 0.5 & 1000 & 498 & 160.3 & 55.1 & $<2.2 \times 10^{-16}$ \\
\hline & 0.08 & & & 498 & 160.3 & 53.98 & $<2.2 \times 10^{-16}$ \\
\hline & 0.01 & & & 498 & 160.3 & 53.49 & $<2.2 \times 10^{-16}$ \\
\hline & 0.05 & & 200 & 498 & 482.27 & 129.2 & $<2.2 \times 10^{-16}$ \\
\hline & & & 10000 & 498 & 63.06 & 25.14 & $<2.2 \times 10^{-16}$ \\
\hline & & 0.2 & 1000 & 498 & 53.57 & 29.74 & $<2.2 \times 10^{-16}$ \\
\hline & & 0.8 & & 498 & 235.86 & 70.17 & $<2.2 \times 10^{-16}$ \\
\hline & & 0.95 & & 498 & 251.77 & 79.7 & $<2.2 \times 10^{-16}$ \\
\hline \multirow[t]{6}{*}{16} & 0.01 & 0.5 & 1000 & 467 & 2476.75 & 1858.21 & $4.2 \times 10^{-6}$ \\
\hline & 0.05 & & & 462 & 2437.04 & 1886.83 & 0.0001328 \\
\hline & 0.025 & & 200 & 248 & 3381.98 & 3389.867 & 0.778 \\
\hline & & & 10000 & 500 & 548.65 & 392.58 & $1.5 \times 10^{-14}$ \\
\hline & & 0.2 & 1000 & 500 & 225.74 & 187.78 & $5.4 \times 10^{-7}$ \\
\hline & & 0.8 & & $283^{a}$ & 4172.47 & 3494.55 & 0.00204 \\
\hline
\end{tabular}

The number of generations that a population takes to 'discover' a circuit in a new genotype network is significantly lower when we allow plasticity $\left(t_{*}\right.$, plast $<t_{*}$, control), according to a Wilcoxon signed-rank test.

The value of $d$ is that of the old genotype network. We analyzed 500 pairs of evolving populations for each combination of $N, c$ and $d$. We discarded population pairs in which any of the populations had not reached the new genotype network by the end of the simulation $\left(t=10^{4}\right)$. Thus, our actual sample size was lower than 500 populations. The remaining parameter values are as follows: $d=0.25 ; \mu=0.5$. $c \approx 0.4$ when $N=8$ and it equals 0.2 when $N=16$.

${ }^{a}$ We analyzed 1500 pairs of evolving populations for the simulations that we report in this row, but the populations found the new genotype network only in 283 cases. 
Table 7 Plasticity slows down the frequency increase of circuits in a new optimal genotype network

\begin{tabular}{|c|c|c|c|c|c|c|}
\hline$N$ & $c$ & $d$ & $\begin{array}{l}\text { Sample } \\
\text { size }\end{array}$ & $\begin{array}{c}\text { Mean } t_{0.25} \\
\text { control }\end{array}$ & $\begin{array}{c}\text { Mean } t_{0.25} \text { plast } \\
\text { pla }\end{array}$ & $p$-value \\
\hline \multirow[t]{6}{*}{8} & 0.4 & 0.125 & 498 & 27.55 & 46.72 & $<2.2 \times 10^{-16}$ \\
\hline & & 0.25 & 498 & 30.69 & 45.91 & $<2.2 \times 10^{-16}$ \\
\hline & & 0.5 & 497 & 28.01 & 45.63 & $<2.2 \times 10^{-16}$ \\
\hline & 0.3 & 0.125 & 495 & 18.8 & 26.49 & $<2.2 \times 10^{-16}$ \\
\hline & & 0.25 & 498 & 19.25 & 25.86 & $<2.2 \times 10^{-16}$ \\
\hline & & 0.5 & 498 & 26.44 & 25.99 & $<2.2 \times 10^{-16}$ \\
\hline \multirow[t]{6}{*}{16} & 0.25 & 0.125 & 413 & 41.61 & 106.96 & $6.9 \times 10^{-14}$ \\
\hline & & 0.25 & 431 & 35.66 & 138.98 & $<2.2 \times 10^{-16}$ \\
\hline & & 0.5 & 418 & 49.76 & 162.98 & $<2.2 \times 10^{-16}$ \\
\hline & 0.2 & 0.125 & 462 & 43.4 & 143.45 & $<2.2 \times 10^{-16}$ \\
\hline & & 0.25 & 466 & 36.48 & 133.72 & $<2.2 \times 10^{-16}$ \\
\hline & & 0.5 & 471 & 42.01 & 120.97 & $<2.2 \times 10^{-16}$ \\
\hline 20 & 0.2 & 0.25 & 152 & 35.45 & 103.8 & $4.4 \times 10^{-10}$ \\
\hline
\end{tabular}

The number of generations that a population needs to increase the fraction of its circuits in the new genotype network to 25 percent is significantly higher with plasticity, i.e., $t_{0.25 \text {,plast }}>t_{0.25 \text {, control }}$ according to a Wilcoxon signedrank test.

The value of $d$ is that of the old genotype network. We analyzed 500 pairs of evolving populations for each combination of $N, c$ and $d$. We discarded population pairs in which any of the populations never had $25 \%$ of its circuits in the new genotype network by the end of the simulation $\left(t=10^{4}\right)$. Thus, our actual sample size was lower than 500 populations. The probability $\alpha$ of geneactivity perturbation in $s_{0}$ equaled 0.05 per gene when $N=8,0.025$ when $N=16$, and 0.02 when $N=20$. Population size $M=1000 ; \mu=0.5 ; \omega^{\text {native }}=0.5$.

phenotypes they might adopt. In other words, it allows us to determine how gene expression phenotypes are organized in circuit genotype space. This organization has several properties that facilitate the discovery of a new phenotype through plasticity and its genetic stabilization (Figure 6). First, many circuits show plastic phenotypes, which helps them explore many more novel phenotypes than mutation alone could. Second, many circuits that can produce a new phenotype $P_{\text {new }}$ through plasticity are neighbors of the genotype network of $P_{\text {new }}$. They thus allow mutational access to a genotype whose native phenotype is $P_{\text {new }}$. Third, similar genotypes on the same genotype network often produce the same alternative phenotypes. Fourth, the higher the penetrance of a new phenotype $P_{\text {new }}$ in a genotype $G$ is, the easier it is to reach $P_{\text {new }}$ 's genotype network from $G$. From these observations emerges an important role for plasticity in the discovery and stabilization of novel phenotypes (Figure 1): Genotypes that produce a new phenotype through plasticity (i.e. after non-genetic perturbations) may frequently be intermediates in evolutionary paths towards genotypes where that phenotype is the 'native' phenotype.

Evolutionary simulations show that plasticity can indeed accelerate evolutionary adaptation. In these simulations we determined the time a population needs

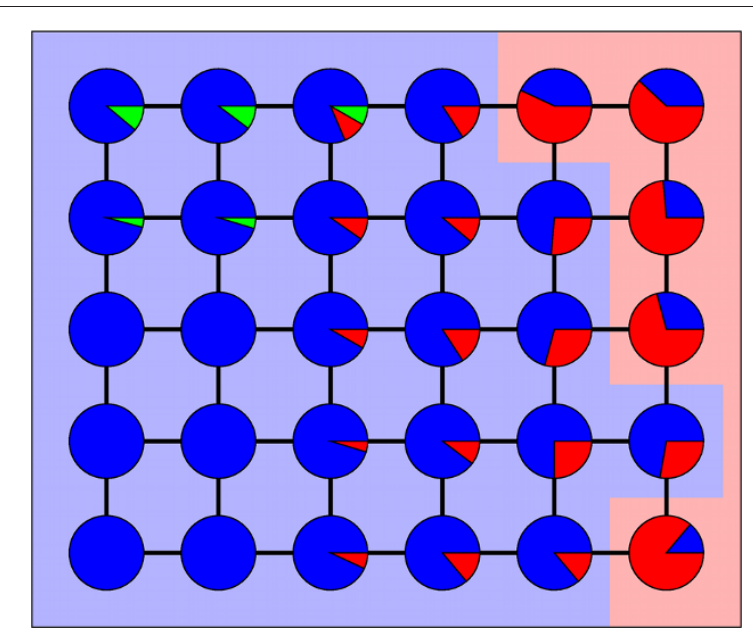

Figure 6 The organization of gene circuit genotype space favors plasticity-mediated adaptive evolution. Shapes and colors have the same meanings in the diagram as in Figure 1. Starting from the blue genotype network, it is easier to find a genotype that produces a red alternative phenotype than finding the red genotype network. Many genotypes that produce the red alternative phenotype have direct mutational access to the red genotype network. Such genotypes are neighbors of other genotypes that produce the same alternative phenotype. Among genotypes that produce the red alternative phenotype, a high penetrance is associated to easier access to the red genotype network.

to 'discover' the genotype network of an arbitrary novel and adaptive phenotype. This time is significantly lower when phenotypes are plastic than when they are not.

At the same time, plasticity causes a small reduction in the rate at which the frequency of circuits in a new genotype network increases. The reason is straightforward. In the absence of plasticity, genotypes in the new genotype network always produce the new, best adapted phenotype. With plasticity, the same genotypes occasionally produce other, suboptimal phenotypes: These genotypes are thus overall less well adapted than in the absence of plasticity, which slows their ascent in the population. The resulting delay is similar to that predicted by previous theoretical work $[22,29,73,74]$ that showed smaller selection differentials in populations with plasticity. These smaller differentials can result from either the different fitness effects of the distinct phenotypes that a genotype may produce $[22,74]$, or from alleles that are visible to selection only under certain environmental or genetic conditions [29,73]. At least in our study system, this delay is insufficient to cancel out the acceleration in finding a new genotype network. Plasticity accelerates the discovery of the new genotype network to a much greater extent than it slows down the 'conquest' of this network by a population (Tables 5,7 ).

In our system, the positive role of plasticity in the origin and stabilization of new phenotypes is closely tied to 
the existence of large genotype networks. Such networks allow access to very distant regions in genotype space, while preserving an existing native phenotype. And because the spectrum of alternative phenotypes in these regions is not the same, genotype networks facilitate the exploration of many novel phenotypes through plasticity.

Early studies, albeit based on an unrealistic genotypephenotype map, suggested that plasticity could improve the efficiency of an evolutionary search by smoothing fitness landscapes [75]. Ancel and Fontana asked related questions with a more realistic genotype-phenotype map: the folding of RNA molecules [22]. These authors showed that plasticity does not accelerate adaptive evolution in RNA structures. The reason is that RNA molecules lack the first of our four properties: It is not much easier to discover a novel alternative phenotype than to discover this phenotype's genotype network. This occurs because finding an RNA genotype capable of producing two specific secondary structure phenotypes severely constrains the allowed base-pairings in each RNA structure [22].

In contrast to the RNA system, plasticity has a positive role for evolutionary adaptation in our regulatory circuits. Thus, the role of plasticity may depend on the level of biological organization one focuses on. We note that much of the empirical evidence supporting plasticity's importance for adaptation comes from morphological traits, such as head size in snakes, and developmental decisions, such as those involving spore formation in bacteria [30-35,42,46]. In this regard, it is significant that gene regulatory circuits are central to shape these kinds of traits. It may thus not be a coincidence that plasticity can facilitate evolutionary transitions in these traits. Our modeling results suggest that future experimental analyses will produce observations similar to existing experiments and similar to our observations wherever regulatory circuits are involved in adaptation.

Transcriptional regulation circuits, while important, are not the only systems in which change can lead to novel traits that confer a fitness advantage. Changes in mechanical, biochemical or geometrical cell and tissue properties, in cell-cell signaling, in hormonal communication between distant parts of the organism, or even in behavioral traits can lead to new, potentially adaptive, phenotypes. Whether plasticity facilitates adaptive evolution in all these traits and systems is unknown. To answer this question will require experimental studies of evolution in individual, mechanistically well-understood systems associated with innovation, combined with computational work like ours that reveals generic principles of evolution for a system class. In addition, the changes that constitute an adaptive novelty frequently span several levels of biological organization and multiple kinds of systems. New theoretical and empirical approaches are needed to ask how such different levels of biological organization interact in producing phenotypic variation. Such approaches will be important to understand the evolutionary origin of complex adaptations and the role of plasticity beyond individual case studies.

\section{Conclusions}

Our results predict that phenotypic plasticity will have an important role in adaptive evolution that involves changes in gene activity. The fundamental reason is that genotypes that produce occasionally a beneficial phenotype (and thus have a selective advantage) give more easily rise to genotypes where that same phenotype is more strongly genetically determined. New adaptive phenotypes may frequently arise first as alternative phenotypes, induced by non-genetic perturbations, and then be genetically stabilized by selection. Our work thus suggests a widespread relationship between phenotypic plasticity and adaptive evolution in gene activity phenotypes.

\section{Methods}

Throughout this work, we chose new non-zero entries in a circuit's interaction matrix from a normal distribution with mean zero and standard deviation one. Also throughout this work, we disregarded the phenotypes of circuits that attained no stable fixed-point activity pattern, as in previous studies [11].

\section{Sampling of random genotypes in a genotype network}

To sample genotypes, we first used previously established procedures to identify a circuit genotype that produces a certain gene activity phenotype $s_{\infty}^{\text {native }}$ from an initial condition $\mathrm{s}_{0}$ [7]. Then, we imposed $10^{4}$ random mutations that preserve the production of $s_{\infty}^{\text {native from }}$ $s_{0}$, to erase any trace of the initial genotype. Each mutation was picked at random from many possible alternatives (see 'Phenotypes accessible through mutation' below). The genotype obtained in this manner is the first in our sample. To sample additional genotypes in the same genotype network, we subjected the genotype to a series of $5 m_{+}$mutations that we required to preserve $s_{\infty}^{\text {native }}$. Here, $m_{+}$stands for the maximum number of interactions allowed in a circuit. This procedure allowed us to avoid correlations between consecutively sampled genotypes.

\section{Phenotypes accessible through perturbations in the initial condition $s_{0}$}

For a circuit genotype that attains $s_{\infty}^{\text {native }}$ when its developmental dynamics start from $s_{0}$, we determined the 
phenotype of the $N$ possible single gene-activity modifications of $s_{0}$. Wherever we explored the effects of double gene perturbations of the initial condition, we followed the same procedure, but for all $N(N-1) / 2$ possible two-gene perturbations.

\section{Phenotypes accessible through mutation}

To generate all neighbors of a circuit, we examined each entry $a_{i j}$ in the matrix $\mathbf{A}$ of the circuit, and distinguished the following cases: if $a_{i j} \neq 0$ we determined the phenotype of two mutants, one in which the circuit loses an interaction $\left(a_{i j}=0\right)$, and one in which the value of $a_{i j}$ changes but we force its sign to remain unaltered; if $a_{i j}$ $=0$ we obtain the phenotype of two mutants, one in which $a_{i j}>0$, and one in which $a_{i j}<0$. Whenever we required a value of $a_{i j}$ to be either smaller or greater than zero, we forced its sign as needed. To find the phenotype of a mutant circuit we followed the developmental dynamics (Eq. 1) using the unperturbed $s_{0}$ activity pattern as initial condition. We only determined the phenotype of mutants whose number of interactions was equal or smaller than a pre-specified value $m$ and equal or greater than $m-5$. This allowed us to keep interaction density $c$ in a narrow interval, in order to explore the effect of variations on $c$.

\section{Overlap between $P_{\mu}$ and $P_{s_{0}}$}

We used the index $C=\frac{\left|P_{\mu} \cap P_{s_{0}}\right|}{\min \left(\left|P_{\mu}\right|,\left|P_{s_{0}}\right|\right)}$ to assess the similarity between the set of phenotypes that a genotype $G$ produces after single mutations $\left(P_{\mu}\right)$ and the set of phenotypes that $G$ produces after perturbations in the initial condition $\left(P_{s_{0}}\right)$. We obtained values of $C$ for each of $10^{4}$ genotypes in a pre-determined genotype network. Throughout this sampling, we built two lists of phenotypes, $L_{\mu}$ and $L_{s_{0}}$. We kept in either $L_{\mu}$ or $L_{s_{0}}$ the phenotypes that genotypes in the sample could access through either mutation or perturbations in $s_{0}$, respectively. Then, we determined for each genotype a second index $C^{\text {rand }}$, that measures the similarity between $P_{\mu}$ and $P_{s_{0}}$ expected by chance alone. We calculated $C^{r a n d}$ as we calculated $C$, but from randomized phenotype sets. specifically, we randomized a genotype's $P_{\mu}$ $\left(P_{s_{0}}\right)$ by replacing each phenotype in this set by a gene activity pattern picked at random from $L_{\mu}\left(L_{s_{0}}\right)$. We did not allow any phenotype to appear more than once in any one randomized set. Among the $10^{4}$ genotypes that we sampled, we discarded those in which either $P_{\mu}$ or $P_{s_{0}}$ was empty.

Shortest mutational distance to a new genotype network We picked a genotype $G$ at random from the genotype network of a pre-specified phenotype $s_{\infty}^{\text {native }}$. We selected at random one of G's alternative phenotypes, and called it $s_{\infty}^{\text {new }}$. We created $10^{3}$ copies of $G$, and changed each copy's regulatory interactions in a sequence of mutational steps. None of the steps was

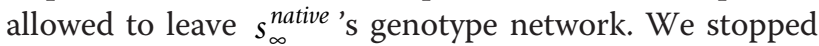
the sequence as soon as one of the circuits was a neigh-

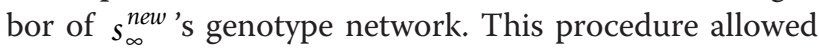
us to estimate an upper-bound for the minimum number of mutations required to reach $s_{\infty}^{\text {new }}$ 's genotype network from $G$.

\section{Evolutionary simulations}

We established a population of $M=10^{3}$ identical circuits. Such circuits produce a phenotype $s_{\infty}^{\text {native }}$ from an initial state $s_{0}$. We also picked a gene activity pattern where each gene's activity state was picked at random. This activity pattern represents the new optimal phenotype $s_{\infty}^{\text {new }}$ and it is different from the native phenotype $s_{\infty}^{\text {native }}$. We let the population evolve through repeated cycles ('generations') of mutation and selection implemented as follows.

To assess a circuit's phenotype and fitness, we first assigned an initial condition to the circuit by changing the activity state of each gene in the unperturbed initial condition $s_{0}$ with a probability $\alpha$. We used this perturbed initial condition to start the gene activity dynamics dictated by the circuit's matrix A (Eq. 1). The resulting stable (fixed-point) activity pattern $s_{\infty}$ is the circuit's phenotype. We disregarded circuits that did not produce a fixed-point equilibrium state. If a circuit's phenotype $s_{\infty}$ was equal to $s_{\infty}^{\text {new }}$, then we assigned to it a fitness $\omega=\omega^{\text {new }}=1$. Otherwise, we set its fitness $\omega$ equal to the maximum of either $\left(1-y_{\text {new }} / \mathrm{N}\right)^{5}$ or $\omega^{\text {native }}$ $\left(1-y_{\text {native }} \mathrm{N}\right)^{5}$, where $y_{\text {new }}$ equals the number of gene activity differences between the phenotype $s_{\infty}$ and $s_{\infty}^{\text {new }}$, and $y_{\text {native }}$ equals the number of gene activity differences between $s_{\infty}$ and $s_{\infty}^{\text {native }}$. This procedure ensures a steep decrease in fitness for any deviations from gene expression states $s_{\infty}^{\text {new }}$ or $s_{\infty}^{\text {native }}$.

We chose the value of $\alpha$ so that no gene activity was perturbed in the majority of circuits. Unless indicated otherwise, $\alpha=0.02$ when $N=20, \alpha=0.025$ when $N=$ 16 and $\alpha=0.05$ when $N=8$. To study evolutionary dynamics without plasticity, we set $\alpha$ equal to zero in control populations. In these populations, circuits always start their developmental dynamics from $s_{0}$.

At each generation, we sampled individuals from the current population with replacement. For every sampled genotype $G$, we subjected with probability $\mu$ a copy of $G$ to mutation, thus picking one of its (mutational) neighbors at random (see 'Phenotypes accessible through mutation' above). We allowed such a new circuit in the new generation with a probability equal to its fitness. In other words, we considered fitness as survival probability. We 
continued sampling until $M$ new individuals constituted the population for the next generation. We stopped evolution after either at least one quarter of the population resided in the new genotype network, or after $10^{4}$ generations, whichever came first. For all simulations, $\omega^{\text {native }}=$ $0.5, M=10^{3}, \mu=0.5$, unless indicated otherwise.

\section{Additional material}

Additional file 1: Figure S1. Gene circuits exploring a genotype network find genotypes with new alternative phenotypes faster than they find new genotype networks, for different parameter combinations.

Additional file 2: Figure S2. Mutations and perturbations in the initial condition $s_{0}$ produce the same phenotypes more often than expected by chance.

Additional file 3: Analysis S1. Genetic distance to a genotype network decreases with increasing phenotypic penetrance, for alternative phenotypes produced after two-gene perturbations in the initial condition.

Additional file 4: Analysis S2. High penetrance increases the number of mutational paths to a new genotype network.

Additional file 5: Analysis S3. Mutations that increase in penetrance facilitate access to a new genotype network.

\section{Acknowledgements}

We thank three anonymous reviewers for suggestions and comments that improved this manuscript greatly. The research leading to these results has received funding from the European Community's Seventh Framework Programme FP7/2007-2013 under grant agreement n PIIF-GA-2008-220274. AW acknowledges support through SNF grants 315200-116814, 315200119697 and 315230-129708 as well as through the YeastX project of SystemsX.ch, and the University Priority Research Program in systems biology at the University of Zurich.

\section{Author details}

${ }^{1}$ University of Zurich, Dept. of Biochemistry, Bldg. Y27 Winterthurerstrasse $190 \mathrm{CH}-8057$ Zurich, Switzerland. ${ }^{2}$ The Swiss Institute of Bioinformatics. Quartier Sorge, Batiment Genopode, 1015 Lausanne, Switzerland. ${ }^{3}$ INRA, UMR 0320/UMR 8120 Génétique Végétale, F-91190 Gif-sur- Yvette, France. ${ }^{4}$ The Santa Fe Institute, 1399 Hyde Park Road, Santa Fe, NM 87501, USA.

\section{Authors' contributions}

CE-S designed most of the analyses, wrote program scripts, carried out the analyses, interpreted the results and drafted the manuscript. OCM contributed program codes, designed some of the analyses, and drafted the manuscript. AW designed some of the analyses, interpreted the results and drafted the manuscript. All authors read and approved the final manuscript.

Received: 19 August 2010 Accepted: 6 January 2011

Published: 6 January 2011

\section{References}

1. Wagner GP, Altenberg L: Complex adaptations and the evolution of evolvability. Evolution 1996, 50:967-976.

2. Rechenberg I: Evolutionsstrategie Stuttgart: Frommann-Holzboog; 1973.

3. Pigliucci M: Is evolvability evolvable? Nat Rev Genet 2008, 9:75-82.

4. Lipman DJ, Wilbur WJ: Modelling neutral and selective evolution of protein folding. Proc R SOC B 1991, 245(1312):7-11

5. Schuster $P$, Fontana W, Stadler PF, Hofacker IL: From sequences to shapes and back: a case study in RNA secondary structures. Proc R Soc B 1994, 255:279-284.

6. Schultes EA, Bartel DP: One sequence, two ribozymes: implications for the emergence of new ribozyme folds. Science 2000, 289:448-452.
7. Ciliberti S, Martin OC, Wagner A: Robustness can evolve gradually in complex regulatory gene networks with varying topology. PLoS Comput Biol 2007, 3(2):e15.

8. Rodrigues JFM, Wagner A: Evolutionary plasticity and innovations in complex metabolic reaction networks. PLoS Comput Biol 2009, 5:e1000613.

9. Fontana W: Modelling 'evo-devo' with RNA. BioEssays 2002, 24:1164-1177.

10. Schuster $P$, Fontana $W$ : Chance and necessity in evolution: lessons from RNA. Physica D 1999, 133:427-452.

11. Ciliberti S, Martin OC, Wagner A: Innovation and robustness in complex regulatory gene networks. Proc Natl Acad Sci USA 2007, 104(34):13591-13596.

12. Ferrada $E$, Wagner $A$ : Protein robustness promotes evolutionary innovations on large evolutionary time-scales. Proc $R$ Soc B 2008, 275:1595-1602.

13. Wagner A: Neutralism and selectionism: a network-based reconciliation. Nat Rev Genet 2008, 9(12):965-974.

14. Wagner A: Robustness and evolvability in living systems Princeton Studies in Complexity, Princeton, NJ: Princeton University Press; 2005.

15. Eyre-Walker A, Keightley PD: The distribution of fitness effects of new mutations. Nat Rev Genet 2007, 8:610-618.

16. Orr HA: Fitness and its role in evolutionary genetics. Nat Rev Genet 2009, 10:531-539.

17. West-Eberhard MJ: Developmental Plasticity and Evolution New York: Oxford University Press; 2003.

18. Pigliucci M, Murren CJ: Genetic assimilation and a possible evolutionary paradox: can macroevolution sometimes be so fast as to pass us by? Evolution 2003, 57:1455-1464.

19. Gilbert SF, Epel D: Ecological Developmental Biology: Integrating Epigenetics, Medicine, and Evolution Sunderland, MA, USA: Sinauer Associates; 2008.

20. Rutherford SL: Between genotype and phenotype: protein chaperones and evolvability. Nat Rev Genet 2003, 4:263-274.

21. Prusiner SB: Prions. Proc Natl Acad Sci USA 1998, 95:13363-13383.

22. Ancel LW, Fontana W: Plasticity, evolvability, and modularity in RNA. J Exp Zool (Mol Dev Biol) 2000, 288:242-283.

23. Henkin TM: Riboswitch RNAs: Using RNA to sense cellular metabolism. Genes Dev 2008, 22:3383-3390.

24. McAdams HH, Arkin A: Stochastic mechanisms in gene expression. Proc Natl Acad Sci USA 1997, 94:814-819.

25. Elowitz MB, Levine AJ, Siggia ED, Swain PS: Stochastic gene expression in a single cell. Science 2002, 297:1183-1186.

26. Raj A, Rifkin SA, Andersen E, van Oudenaarden A: Variability in gene expression underlies incomplete penetrance. Nature 2010, 463(7283):913-918.

27. Gibson G: The environmental contribution to gene expression profiles. Nat Rev Genet 2008, 9(8):575-581.

28. Hodgins-Davis A, Townsend JP: Evolving gene expression: from $G$ to $E$ to GxE. Trends Ecol Evol 2009, 24:649-658.

29. Snell-Rood EC, Dyken JDV, Cruickshank T, Wade MJ, Moczek AP: Toward a population genetic framework of developmental evolution: the costs, limits, and consequences of phenotypic plasticity. BioEssays 2010, 32:71-81.

30. Palmer AR: Symmetry breaking and the evolution of development. Science 2004, 306(5697):828-833.

31. Ledon-Rettig CC, Pfennig DW, Nascone-Yoder N: Ancestral variation and the potential for genetic accommodation in larval amphibians: implications for the evolution of novel feeding strategies. Evol Dev 2008, 10(3):316-325.

32. Gomez-Mestre I, Buchholz DR: Developmental plasticity mirrors differences among taxa in spadefoot toads linking plasticity and diversity. Proc Natl Acad Sci USA 2006, 103:19021-19026.

33. Chapman LJ, Galis F, Shinn J: Phenotypic plasticity and the possible role of genetic assimilation: Hypoxia-induced trade-offs in the morphological traits of an African cichlid. Ecol Lett 2000, 3(5):387-393.

34. Scoville AG, Pfrender ME: Phenotypic plasticity facilitates recurrent rapid adaptation to introduced predators. Proc Natl Acad Sci USA 2010, 107:4260-4263.

35. Aubret F, Shine R: Genetic assimilation and the postcolonization erosion of phenotypic plasticity in island tiger snakes. Curr Biol 2009, 19:1932-1936.

36. Wund MA, Baker JA, Clancy B, Golub JL, Foster SA: A test of the "flexible stem" model of evolution: ancestral plasticity, genetic accommodation, 
and morphological divergence in the threespine stickleback radiation. Am Nat 2008, 172:449-462.

37. Suzuki Y, Nijhout HF: Evolution of a polyphenism by genetic accomodation. Science 2006, 311:650-652.

38. Rutherford SL, Lindquist S: Hsp90 as a capacitor for morphological evolution. Nature 1998, 396:336-342.

39. Moczek AP: Developmental capacitance, genetic accommodation, and adaptive evolution. Evol Dev 2007, 9:299-305.

40. Pigliucci M, Murren CJ, Schlichting CD: Phenotypic plasticity and evolution by genetic assimilation. J Exp Biol 2006, 209:2362-2367.

41. Newman SA, Forgacs G, Müller GB: Before programs: the physical origination of multicellular forms. Int J Dev Biol 2006, 50:289-299.

42. Eldar A, Chary VK, Xenopoulos P, Fontes ME, Losón OC, Dworkin J, Piggot PJ, Elowitz MB: Partial penetrance facilitates developmental evolution in bacteria. Nature 2009, 460:510-514.

43. Moczek AP: On the origins of novelty in development and evolution. BioEssays 2008, 30(5):432-447.

44. Pfennig DW, Wund MA, Snell-Rood EC, Cruickshank T, Schlichting CD, Moczek AP: Phenotypic plasticity's impacts on diversification and speciation. Trends Ecol Evol 2010, 25(8):459-467.

45. Waddington $\mathrm{CH}$ : Canalization of development and the inheritance of acquired characters. Nature 1942, 150:563-565.

46. Waddington $\mathrm{CH}$ : Genetic assimilation of an acquired character. Evolution 1953, 7:118-126.

47. Schmalhausen II: Factors of Evolution. The theory of stabilizing selection Chicago, IL: University of Chicago Press; 1949.

48. Baldwin JM: A new factor in evolution. Am Nat 1896, 30:441-451, 536-553.

49. Waddington $\mathrm{CH}$ : Genetic assimilation of the Bithorax phenotype. Evolution 1956, 10:1-13.

50. Orr HA: An evolutionary dead end? Science 1999, 285:343-344

51. de Jong G, Crozier RH: A flexible theory of evolution. Nature 2003, 424:16-17.

52. Braendle C, Flatt T: A role for genetic accommodation in evolution? BioEssays 2006, 28:868-873.

53. Hall BK: Organic selection: Proximate environmental effects on the evolution of morphology and behaviour. Biol Philos 2001, 16:215-237.

54. Davidson EH, Levine MS: Properties of developmental gene regulatory networks. Proc Natl Acad Sci USA 2008, 105:20063-20066.

55. Erwin DH, Davidson EH: The evolution of hierarchical gene regulatory networks. Nat Rev Genet 2009, 10:141-148.

56. Shubin N, Tabin C, Carroll S: Deep homology and the origins of evolutionary novelty. Nature 2009, 457:818-823.

57. Moczek AP, Rose DJ: Differential recruitment of limb patterning genes during development and diversification of beetle horns. Proc Natl Acad Sci USA 2009, 106:8992-8997.

58. Fraser GJ, Hulsey CD, Bloomquist RF, Uyesugi K, Manley NR, Streelman JT: An ancient gene network is co-opted for teeth on old and new jaws. PLoS Biol 2009, 7:e1000031.

59. Gao F, Davidson EH: Transfer of a large gene regulatory apparatus to a new developmental address in echinoid evolution. Proc Natl Acad Sci USA 2008, 105:6091-6096

60. Martin OC, Wagner A: Effects of recombination on complex regulatory circuits. Genetics 2009, 183:673-684.

61. Espinosa-Soto C, Wagner A: Specialization can drive the evolution of modularity. PLoS Comput Biol 2010, 6:e1000719.

62. Azevedo RBR, Lohaus R, Srinivasan S, Dang KK, Burch CL: Sexual reproduction selects for robustness and negative epistasis in artificial gene networks. Nature 2006, 440:87-90.

63. Bergman A, Siegal ML: Evolutionary capacitance as a general feature of complex gene networks. Nature 2003, 424:549-552.

64. Siegal ML, Bergman A: Waddington's canalization revisited: Developmental stability and evolution. Proc Natl Acad Sci USA 2002, 99:10528-10532.

65. Draghi J, Wagner GP: The evolutionary dynamics of evolvability in a gene network model. J Evol Biol 2009, 22:599-611.

66. Salazar-Ciudad I, Newman S, Solé RV: Phenotypic and dynamical transitions in model genetic networks I. Emergence of patterns and genotype-phenotype relationships. Evol Dev 2001, 3:84-94.

67. Mjolsness E, Sharp DH, Reinitz J: A connectionist model of development. J Theor Biol 1991, 152:429-453.
68. Mendoza L, Alvarez-Buylla ER: Dynamics of the genetic regulatory network of Arabidopsis thaliana flower morphogenesis. J Theor Biol 1998, 193:307-319.

69. Macdonald PM, Ingham P, Struhl G: Isolation, structure, and expression of even-skipped: a second pair-rule gene of Drosophila containing a homeo box. Cell 1986, 47:721-734.

70. Harding K, Rushlow C, Doyle HJ, Hoey T, Levine M: Cross-regulatory interactions among pair-rule genes in Drosophila. Science 1986, 233:953-959.

71. Carey CC, Gorman KF, Rutherford S: Modularity and intrinsic evolvability of Hsp90-buffered change. PLOS ONE 2008, 1:e76.

72. Wagner GP, Chiu CH, Hansen TF: Is Hsp90 a regulator of evolvability? 」 Exp Zool (Mol Dev Evol) 1999, 285:116-118.

73. Van Dyken JD, Wade MJ: The genetic signature of conditional expression. Genetics 2010, 184(2):557-570.

74. Ancel LW: Undermining the Baldwin expediting effect: does phenotypic plasticity accelerate evolution? Theor Popul Biol 2000, 58:307-319.

75. Hinton GE, Nowlan SJ: How learning can guide evolution. Complex Systems 1987, 1:495-502.

76. Hollander M, Wolfe DA: Nonparametric Statistical Methods New York, USA: John Wiley \& Sons; 1973.

doi:10.1186/1471-2148-11-5

Cite this article as: Espinosa-Soto et al:: Phenotypic plasticity can

facilitate adaptive evolution in gene regulatory circuits. BMC Evolutionary Biology 2011 11:5.

\section{Submit your next manuscript to BioMed Central and take full advantage of:}

- Convenient online submission

- Thorough peer review

- No space constraints or color figure charges

- Immediate publication on acceptance

- Inclusion in PubMed, CAS, Scopus and Google Scholar

- Research which is freely available for redistribution 